\title{
Atributos químicos de um Neossolo Quartzarênico sob capim- marandu adubado com resíduo líquido de frigorífico
}

\section{Chemical properties in Entisol under pasture grass marandu fertilizer of liquid waste of bovine slaughter}

\author{
Sabino Pereira da Silva Neto ${ }^{1}$; Antonio Clementino dos Santos ${ }^{2 *}$; José Expedito \\ Cavalcante da Silva ${ }^{2}$; Valdinéia Patrícia Dim; Aridouglas dos Santos Araújo ${ }^{1}$
}

\section{Resumo}

Este experimento foi realizado com o objetivo de avaliar o efeito da aplicação de resíduo líquido de frigorífico (RLF) sobre as características químicas de um Neossolo Quartzarênico Órtico típico sob pastagem de capim-Marandu. Foram aplicadas quatro doses de RLF $\left(0 ; 37,5 ; 75 ; 112,5 \mathrm{~m}^{3}\right.$ ha $^{-}$ $\left.{ }^{1}\right)$. O delineamento experimental utilizado foi em blocos inteiramente casualizados. Foram coletadas amostras de solo nas profundidades de 0-10, 10-20, 20-30, 30-40 cm, em cinco repetições, nas quais foram analisados $\mathrm{pH}, \mathrm{P}$ e K $\mathrm{K}^{+}$disponíveis, $\mathrm{Ca}^{2+}, \mathrm{Mg}^{2+}$ e $\mathrm{Al}^{3+}$ trocáveis, $\mathrm{CTC}_{\mathrm{pH} 7,0}, \mathrm{CTC}_{\text {efetiva, }}$, teores de matéria orgânica e acidez potencial $(\mathrm{H}+\mathrm{Al})$ após 90 dias da aplicação do resíduo líquido. Foi calculada a saturação por bases trocáveis (V\%) e por alumínio (m\%). A aplicação de RLF nas doses de 75 e 112,5 $\mathrm{m}^{3} \mathrm{ha}^{-1}$ reduziram o $\mathrm{pH}$ e os teores de $\mathrm{Al}^{3+}$ e aumentaram a $\mathrm{V} \%$ na camada de $0-10 \mathrm{~cm}$ e a $\mathrm{CTC}_{\text {efetiva }}$ na camada de 10-20 cm. Os teores de matéria orgânica não foram influenciados pela aplicação do RLF. Os teores de $\mathrm{Mg}^{2+}$ aumentaram na camada 30-40 $\mathrm{cm}$ na dose de $112,5 \mathrm{~m}^{3} \mathrm{ha}^{-1}$. Os resultados evidenciam que a aplicação de $37,5 \mathrm{~m}^{3} \mathrm{ha}^{-1}$ aumentou o $\mathrm{pH}$ e os teores de $\mathrm{Ca}^{2+}$ e $\mathrm{K}^{+}$na profundidade de $0-10 \mathrm{~cm}$ e o valor da $\mathrm{CTC}_{\mathrm{pH} 7,0}$ de 0 a $30 \mathrm{~cm}$. O pH, soma de bases, $\mathrm{CTC}_{\text {efetiva }}, \mathrm{CTC}_{\mathrm{pH} 7}$, saturação por bases, $\mathrm{K}^{+}, \mathrm{Ca}^{2+}$, $\mathrm{Mg}^{2+}$ foram influenciados pela profundidade com maiores valores na camada de 0-10 $\mathrm{cm}$ em relação às demais, em todos os tratamentos.

Palavras-chave: Adubação orgânica, Brachiaria brizantha, efluente agroindustrial, química do solo

\begin{abstract}
This study was conducted to evaluate the effect of liquid waste of bovine slaughter (RLF) on the chemical characteristics of a Entisol under pasture grass Marandu. We applied four levels of RLF $(0,37.5,75$, $\left.112.5 \mathrm{~m}^{3} \mathrm{ha}^{-1}\right)$. The experimental design was randomized blocks. The soil samples were collected at $0-10$, 10-20, 20-30, 30-40 cm, deep five replicates, which were analyzed in $\mathrm{pH}$, available $\mathrm{P}$ and $\mathrm{K}^{+}, \mathrm{Ca}^{2+}, \mathrm{Mg}^{2+}$ and $\mathrm{Al}^{3+}, \mathrm{CTC}_{\mathrm{pH} 70}, \mathrm{CTC}_{\text {effective }}$, organic matter content and potential acidity $\left(\mathrm{H}^{+} \mathrm{Al}^{3+}\right)$. We calculated the saturation of exchangeable bases $(\mathrm{V} \%)$ and aluminum $(\mathrm{m} \%)$. The applications of RLF in doses of 75 and $112.5 \mathrm{~m}^{3} \mathrm{ha}^{-1}$ reduced the $\mathrm{pH}$ and level of $\mathrm{Al}^{3+}$. We observed increased of the value the $\mathrm{V} \%$ at $0-10 \mathrm{~cm}$ and $\mathrm{CTC}_{\text {effective }}$ in 10-20 cm. The organic matter content had no significant increases for the implementation of RLF. The $\mathrm{Mg}^{2+}$ increased in the $30-40 \mathrm{~cm}$ layer at $112.5 \mathrm{~m}^{3} \mathrm{ha}^{-1}$. The results suggest that application of $37.5 \mathrm{~m}^{3} \mathrm{ha}^{-1}$ increases the $\mathrm{pH}$ and $\mathrm{Ca}^{2+}$ and $\mathrm{K}^{+}$at a depth of $0-10 \mathrm{~cm}$ and the value of $\mathrm{CTC}_{\mathrm{pH} 7,0}$ to $30 \mathrm{~cm}$. The $\mathrm{pH}$, total bases, $\mathrm{CTC}, \mathrm{CTC}_{\mathrm{pH} 7,0}$, saturation on base, $\mathrm{K}^{+}, \mathrm{Ca}^{2+}, \mathrm{Mg}^{2+}$ were influenced by the depth with higher values in the $0-10 \mathrm{~cm}$ depth compared with other in all the treatments.
\end{abstract}

Key words: Organic fertilization, Brachiaria brizantha, effluent industry meat, soil chemistry

\footnotetext{
${ }^{1}$ Discente(s) de Doutorado(s) do PPG em Ciência Animal Tropical, Centro de Ciência Animal, Universidade Federal do Tocantins, UFT, BR 153, Km 112, Araguaína, TO, CEP 77804-970. E-mail: sabinozootec@yahoo.com.br; valpatdim@hotmail.com; aridouglas_araujo@hotmail.com

${ }^{2}$ Profs. da UFT, Araguaína, TO. Bolsista Produtividade CNPq. E-mail: clementino@uft.edu.br; jecs@uft.edu.br

* Autor para correspondência
} 


\section{Introdução}

A fertilidade dos solos, a adubação e a nutrição são indispensáveis para a manutenção de um sistema de produção eficiente de gramíneas quando se utiliza, por exemplo, a Brachiaria brizantha cv. Marandu como planta forrageira, tendo em vista que a sua produção e composição química são influenciadas por esses fatores.

A produção e a manutenção do crescimento das gramíneas sob pastejo dependem do aporte de nutrientes. No entanto, os Neossolos Quartzarênicos, que abrangem 16,81\% do Tocantins (SANTOS; RABELO, 2008), possuem baixa capacidade de fornecimento de nutrientes o que torna a produção das pastagens, sem a utilização de corretivos e de fertilizantes, bastante aquém do potencial de produção das gramíneas.

$\mathrm{Na}$ agricultura brasileira, nos últimos anos, vem aumentando o uso de fertilizantes orgânicos. Esse interesse na utilização de resíduos orgânicos na produção vegetal está fundamentado no alto teor de carbono, de compostos orgânicos e de nutrientes neles contidos (ABREU JUNIOR et al., 2005).

A indústria frigorífica do Tocantins está crescendo de forma significativa, ocasionando alta concentração de resíduos líquidos orgânicos de forma localizada, o qual possui grande potencial de poluição dos recursos hídricos. No entanto, os resíduos líquidos da indústria de frigoríficos de bovinos contêm percentual de matéria orgânica e de nutrientes (GASI, 1993) que podem ser rapidamente sequestrados pela biomassa vegetal, o que diminuir o risco de prejuízos ambientais. Por isso, a adubação orgânica com resíduo líquido de frigorífico se tornou uma alternativa para que os produtores dessa região possam melhorar as propriedades químicas dos solos.

Diversos trabalhos foram realizados com outras fontes orgânicas em diversas culturas, obtendo-se importantes melhorias nas características químicas dos solos. Entre esses, Silva et al. (2001), Simonete et al. (2003), Rocha, Gonçalves e Moura (2004) e Vieira et al. (2005) indicam como principais ganhos o aumento dos teores de matéria orgânica, dos valores da CTC, do $\mathrm{pH}$ e a disponibilização de macronutrientes, o que aumenta o rendimento das culturas. Entre os principais resíduos líquidos estudados no Brasil como fonte de nutrientes ao solo estão a vinhaça, resíduo do processo de destilação de álcool, e os dejetos líquidos de suínos (GLÓRIA, 1996; CERETTA et al., 2003; ABREU JUNIOR et al., 2005; TEDESCO et al., 2008).

As contribuições e incrementos significativos na produtividade e na qualidade agrícola a partir da aplicação sistêmica de resíduo líquido de frigorífico de bovinos são pouco testadas e pesquisadas em condições de solos com baixos teores de matéria orgânica e pouco férteis. Diante disso, é importante investigar os efeitos da aplicação de resíduo líquido de frigorífico bovino sobre as propriedades químicas dos solos.

O objetivo deste trabalho foi avaliar o efeito da aplicação de doses crescentes de resíduo líquido de frigorífico de bovinos nas propriedades químicas de um Neossolo Quartzarênico Órtico típico sob pastagem de Brachiaria brizantha cv. Marandu.

\section{Material e Métodos}

O experimento foi realizado na fazenda da Escola de Medicina Veterinária e Zootecnia da Universidade Federal do Tocantins (EMVZ-UFT), Campus de Araguaína, no período de janeiro a maio de 2008. A área experimental situa-se nas coordenadas geográficas de latitude $07^{\circ} 13^{\prime} 40^{\prime \prime} \mathrm{S}$ e longitude $48^{\circ} 14^{\prime} 25^{\prime \prime}$ W. Segundo metodologia proposta por Köppen (1948) a região apresenta clima tipo Aw (quente úmido) com temperatura média de $28^{\circ} \mathrm{C}$ e precipitação pluviométrica média de $1800 \mathrm{~mm}$ anuais. As médias e respectivos desvios das temperaturas máxima, mínima e média e a precipitação média durante o experimento, foram de $30,72^{\circ} \mathrm{C} \pm 0,40 ; 21,28^{\circ} \mathrm{C} \pm 0,40 ; 26,09^{\circ} \mathrm{C} \pm 0,23$; $224,46 \pm 92,95 \mathrm{~mm}$, respectivamente. 
O solo da área experimental é classificado como Neossolo Quartzarênico Órtico típico (SANTOS et al., 2006). Antes do estabelecimento do pasto foram realizadas amostragens para fins de caracterização da física (CLAESSEN, 1997) e química do solo nas camadas 0-10, 10-20, 20-30, 30-40 cm, com sonda de amostragem (Tabela 1).

Foi realizado o preparo do solo com uma aração e duas gradagens. Independente do tratamento realizou-se a correção do solo com aplicação de calcário dolomítico com base nos dados da análise de solo, pelo método de saturação de base $\left(\mathrm{V}_{2}=\right.$ 50\%). Foi semeada, a lanço, a cultivar Marandu de Brachiaria brizantha L. com taxa de semeadura de $6 \mathrm{~kg}$ de sementes puras viáveis ha-1 ${ }^{-1}$ A forragem foi coletada respeitando-se intervalo de 35 dias entre as amostragens. Com o auxílio de um quadro de amostragem de $0,5 \mathrm{~m}^{2}(1,0 \times 0,5 \mathrm{~m})$ a forragem foi colhida à altura de $15 \mathrm{~cm}$ do solo e levada para pesagem e posterior secagem em estufa à $65^{\circ} \mathrm{C}$. A gramínea após pré-seca e moída, em peneira de 1 $\mathrm{mm}$, foi analisada quanto aos teores de $\mathrm{N}, \mathrm{P}$ e $\mathrm{K}^{+}$ (EMBRAPA, 1999).

Tabela 1. Características físicas e químicas do resíduo líquido de frigorífico utilizado no experimento.

\begin{tabular}{lrrr}
\hline \multicolumn{1}{c}{ Parâmetros } & $1^{\text {a }}$ Aplicação & $2^{\text {a }}$ Aplicação & $3^{\text {a }}$ Aplicação \\
\hline $\mathrm{pH}$ & 7,01 & 6,21 & 6,24 \\
Nitrogênio amoniacal* & 156,80 & 29,68 & 295,00 \\
Nitrito* & 9,00 & 0,39 & 27,30 \\
Nitrato* & 3,30 & 18,00 & 3,00 \\
Sólidos totais* & $18.887,00$ & $10.982,00$ & $9.688,00$ \\
Sólidos sedimentáveis* & 9,60 & 9,00 & 150,00 \\
Fosfato* & 122,00 & 235,30 & 19,25 \\
Sulfeto* & 16,20 & $<0,01$ & $<1,00$ \\
Oxigênio dissolvido* & 0,70 & 0,00 & 0,00 \\
DBO* (1) & $6.200,00$ & $5.230,00$ & $7.400,00$ \\
DQO* (2) & $16.740,00$ & $22.200,00$ & $15.690,00$ \\
Óleos e graxas* & $4.444,50$ & $3.200,00$ & $1.111,00$ \\
\hline
\end{tabular}

${ }^{*} \mathrm{mg} \mathrm{L}{ }^{-1},{ }^{(1)} \mathrm{DBO}$ : demanda bioquímica de oxigênio, (2) DQO: demanda química de oxigênio.

Fonte: Elaboração dos autores.

O delineamento experimental foi em blocos inteiramente casualizados com quatro tratamentos e cinco repetições, totalizando 20 unidades experimentais de $16 \mathrm{~m}^{2}$ (4x4 m). Foram estudadas doses de resíduo líquido de frigorífico (RLF) de bovinos em função da profundidade do solo, correspondente a $0 ; 37,5 ; 75 ; 112,5 \mathrm{~m}^{3} \mathrm{ha}^{-1}$ divididas em três aplicações. O RLF foi aplicado uniformemente com regradores de jardim. Esse resíduo foi caracterizado conforme metodologia da Apha (1995) (Tabela 2), sendo que esse passou por tratamento primário com a remoção de sólidos sedimentáveis, em suspensão e flotáveis com o auxilio de peneira estática. A $1^{\text {a }}$ aplicação (24/01/2008) do RLF foi realizada na implantação da forrageira, a $2^{\mathrm{a}}(17 / 03 / 2008)$ após o corte de uniformização e a $3^{\mathrm{a}}$ aplicação (21/04/2008) após o primeiro corte da forragem. 
Tabela 2. Características químicas e físicas do Neossolo antes da instalação do experimento.

\begin{tabular}{|c|c|c|c|c|c|c|c|c|c|c|}
\hline Camada & M.O. ${ }^{(1)}$ & $\mathrm{pH}$ & $\mathrm{H}^{+}+\mathrm{Al}^{3+}$ & $\mathrm{P}$ & $\mathrm{K}^{+}$ & $\mathrm{Ca}^{2+}$ & $\mathrm{Mg}^{2+}$ & $\mathrm{SB}^{(2)}$ & $\mathrm{CTC}_{\mathrm{e}}^{(3)}$ & $\mathrm{CTC}^{(4)}$ \\
\hline $\mathrm{Cm}$ & $\mathrm{g} \mathrm{dm}^{-3}$ & $\mathrm{CaCl}_{2}$ & $\mathrm{cmol}_{\mathrm{c}} \mathrm{dm}^{-3}$ & $\mathrm{mg} \mathrm{dm}^{-3}$ & \multicolumn{3}{|c|}{-----cmol dm-3----- } & \multicolumn{3}{|c|}{-----cmol $\mathrm{dm}_{\mathrm{c}}^{-3}$} \\
\hline $0-10$ & 16,10 & $4,06^{2}$ & 2,20 & 0,92 & 0,003 & 0,87 & 0,30 & 1,20 & 1,75 & 3,40 \\
\hline $10-20$ & 10,74 & 4,17 & 1,60 & 0,65 & 0,003 & 0,90 & 0,20 & 1,13 & 1,67 & 2,73 \\
\hline $20-30$ & 9,31 & 4,05 & 1,00 & 0,67 & 0,003 & 0,83 & 0,11 & 0,97 & 1,41 & 1,97 \\
\hline \multirow[t]{3}{*}{$30-40$} & 8,08 & 4,23 & 0,80 & 0,62 & 0,003 & 0,82 & 0,12 & 0,97 & 1,38 & 1,77 \\
\hline & $\mathrm{V}^{(5)}$ & $\mathrm{m}^{(6)}$ & $\mathrm{CE}^{(7)}$ & \multicolumn{2}{|c|}{$\mathrm{Ds}^{(8)}$} & Areia & Silte & Argila & \multicolumn{2}{|c|}{ Clas. Text. ${ }^{(9)}$} \\
\hline & -------- & --------- & ds $\mathrm{m}^{-1}$ & \multicolumn{2}{|c|}{$\mathrm{g} \mathrm{cm}^{-3}$} & & $\mathrm{~g} \mathrm{~kg}^{-1}$ & & \\
\hline $0-10$ & 35,32 & 31,40 & 0,07 & \multicolumn{2}{|c|}{1,55} & 948,5 & 19,0 & 32,5 & \multicolumn{2}{|c|}{ Areia } \\
\hline $10-20$ & 41,37 & 32,36 & 0,06 & \multicolumn{2}{|c|}{1,53} & 937,5 & 27,5 & 35,0 & \multicolumn{2}{|c|}{ Areia } \\
\hline $20-30$ & 49,28 & 31,17 & 0,05 & \multicolumn{2}{|c|}{1,63} & 943,0 & 22,0 & 35,0 & \multicolumn{2}{|c|}{ Areia } \\
\hline $30-40$ & 54,71 & 29,79 & 0,04 & \multicolumn{2}{|c|}{1,57} & 935,0 & 30,0 & 35,0 & \multicolumn{2}{|c|}{ Areia } \\
\hline
\end{tabular}

${ }^{(1)}$ M.O.: matéria orgânica, ${ }^{(2)} \mathrm{SB}$ : soma de bases, ${ }^{(3)} \mathrm{CTC}$ : $\mathrm{CTC}$ : capacidade efetiva de troca de cátions, ${ }^{(4)}$ capacidade de troca de cátions, ${ }^{(5)} \mathrm{V}$ : saturação por bases, ${ }^{(6)} \mathrm{m}$ : saturação por alumínio, ${ }^{(7)} \mathrm{CE}$ : condutividade elétrica, ${ }^{(8)} \mathrm{Ds}$.: densidade do solo, ${ }^{(9)} \mathrm{Clas}$. Text.: classe textural.

Fonte: Elaboração dos autores.

As taxas de aplicações do RLF foram calculas em função da concentração de $\mathrm{N}$ no resíduo em que a soma das aplicações para a dose de $112,5 \mathrm{~m}^{3} \mathrm{ha}^{-1}$ não excedessem a quantidade de $100 \mathrm{~kg} \mathrm{ha}^{-1}$ de $\mathrm{N}$ o que poderia provocar perdas de nitrito, nitrato e amônio por lixiviação devido ao alto volume de RLF aplicado. Assim as doses de $0 ; 37,5 ; 75 ; 112,5$ $\mathrm{m}^{3} \mathrm{ha}^{-1}$ de RLF forneceram $0 ; 20,34 ; 48,68 ; 61,02$ $\mathrm{kg} \mathrm{ha}{ }^{-1}$ de $\mathrm{N}\left(\mathrm{NH}_{4}^{+}+\mathrm{NO}_{2}^{-}+\mathrm{NO}_{3}^{-}\right)$e $0 ; 14,12$; 28,24; 46,36 kg ha-1 de $\mathrm{P}$ na forma de $\mathrm{H}_{2} \mathrm{PO}_{4}^{-}$, respectivamente.

Após 90 dias da $3^{\text {a }}$ aplicação de RLF, foram coletadas aleatoriamente em cada unidade experimental cinco amostras simples de solo para formar uma amostra composta em cada camada (0-10, 10-20, 20-30, 30-40 cm), com sonda de amostragem. As amostras compostas de solo foram secas ao ar e peneiradas em peneira de malha de 2,0 mm obtendo-se amostras de terra fina seca ao ar (TFSA) para as análises químicas (EMBRAPA, 1999). Nas amostras de solo, foram determinados o $\mathrm{pH}$ em $\mathrm{CaCl}_{2}$, os teores de $\mathrm{P}$ e $\mathrm{K}^{+}$extraíveis com Mehlich-1 quantificados por colorimetria e fotometria de chama, respectivamente, os teores de $\mathrm{Ca}^{2+}, \mathrm{Mg}^{2+}$ e o $\mathrm{Al}^{3+}$ trocáveis, extraídos com $\mathrm{KCl} 1 \mathrm{~mol} \mathrm{~L}^{-1}$ e quantificados por método analítico
(EMBRAPA, 1999). Os teores de matéria orgânica e valores de capacidade de trocas de cátions (CTC) a pH 7,0, CTC efetiva, soma de bases trocáveis (SB), porcentagem de saturação por alumínio $(\mathrm{m} \%)$ e porcentagem de saturação por bases (V\%) também foram determinados conforme Embrapa (1999).

Os dados das análises químicas do solo foram submetidos à análise de variância seguida pelo teste de Tukey a $5 \%$ de probabilidade para comparação de médias. Já a produção de matéria seca total e extração de nutrientes pelo capim-marandu foram submetidos à análise de variância e regressão, para verificar a significância do efeito das doses de RLF sobre os atributos avaliados.

\section{Resultados e Discussão}

A aplicação de doses crescentes de resíduo líquido de frigorífico (RLF) alterou as características químicas do solo cultivado com capim-Marandu, havendo interação entre as doses e a profundidade do solo Os maiores valores de $\mathrm{pH}$ foram encontrados na camada de $0-10 \mathrm{~cm}$ na dose de $37,5 \mathrm{~m}^{3} \mathrm{ha}^{-1} \mathrm{e}$ os menores valores nas doses de 75 e $112,5 \mathrm{~m}^{3} \mathrm{ha}^{-1}$ de RLF nessa mesma profundidade (Tabela 3). O maior valor de $\mathrm{pH}$ se deve em principio a menor 
produção de massa seca (MS) total de forragem (Tabela 4) em comparação às maiores doses de RLF o que possivelmente, proporcionou menor absorção de cátions $\left(\mathrm{Ca}^{2+}, \mathrm{Mg}^{2+}\right)$, quando em comparação a testemunha. Resultados semelhantes foram observados por Fonseca (2005) irrigando capim Tifton 85 com efluente secundário de esgoto doméstico.

Tabela 3. Parâmetros químicos do solo em função da profundidade e das doses de resíduo líquido de frigorífico (RLF).

\begin{tabular}{|c|c|c|c|c|c|c|c|c|c|}
\hline Parâmetro & Prof. & 0,00 & 37,50 & 75,00 & 112,50 & $\mathrm{CV}$ & & DMS & \\
\hline \multirow{5}{*}{$=\mathrm{pH}\left(\mathrm{CaCl}_{2}\right)$} & $\mathrm{cm}$ & & ------------- & ${ }^{3} \mathrm{ha}^{-1}----$ & --- & $\%$ & Dose & Prof. & Inter. \\
\hline & $0-10$ & $4,82 \mathrm{aB}$ & $5,04 \mathrm{aA}$ & $4,66 \mathrm{aC}$ & $4,58 \mathrm{aC}$ & \multirow{5}{*}{1,24} & \multirow{4}{*}{0,04} & \multirow{4}{*}{0,04} & \multirow{4}{*}{0,09} \\
\hline & $10-20$ & $4,09 \mathrm{cB}$ & $4,20 \mathrm{bA}$ & $4,11 \mathrm{bcB}$ & $3,90 \mathrm{cC}$ & & & & \\
\hline & $20-30$ & $4,17 \mathrm{bcA}$ & $4,23 \mathrm{bA}$ & $4,19 \mathrm{cA}$ & $3,97 \mathrm{bcB}$ & & & & \\
\hline & $30-40$ & $4,19 \mathrm{bA}$ & $4,17 \mathrm{bAB}$ & $4,10 \mathrm{cBC}$ & $4,05 \mathrm{bC}$ & & & & \\
\hline \multirow{5}{*}{$\mathrm{H}+\mathrm{Al}\left(\mathrm{cmol}_{\mathrm{c}} \mathrm{dm}^{-3}\right)$} & $\mathrm{cm}$ & & & & & & & & \\
\hline & $0-10$ & $2,04 \mathrm{aB}$ & $2,38 \mathrm{aA}$ & $1,32 \mathrm{bD}$ & $1,66 \mathrm{aC}$ & \multirow{4}{*}{6,83} & \multirow{4}{*}{0,08} & \multirow{4}{*}{0,08} & \multirow{4}{*}{0,16} \\
\hline & $10-20$ & $1,15 \mathrm{bC}$ & $1,92 \mathrm{bA}$ & $1,67 \mathrm{aB}$ & $1,57 \mathrm{abB}$ & & & & \\
\hline & $20-30$ & $0,74 \mathrm{cD}$ & $1,59 \mathrm{cA}$ & $1,13 \mathrm{cC}$ & $1,42 \mathrm{bcB}$ & & & & \\
\hline & $30-40$ & $0,85 \mathrm{cC}$ & $1,00 \mathrm{dC}$ & $1,17 \mathrm{bcB}$ & $1,37 \mathrm{cA}$ & & & & \\
\hline \multirow{5}{*}{$\mathrm{Al}^{3+}\left(\mathrm{cmol}_{\mathrm{c}} \mathrm{dm}^{-3}\right)$} & $\mathrm{cm}$ & & & & & \multirow{5}{*}{15,31} & & & \\
\hline & $0-10$ & $0,44 \mathrm{aA}$ & $0,27 \mathrm{bB}$ & $0,19 \mathrm{cBC}$ & $0,17 \mathrm{cC}$ & & \multirow{4}{*}{0,04} & \multirow{4}{*}{0,04} & \multirow{4}{*}{0,08} \\
\hline & $10-20$ & $0,45 \mathrm{aA}$ & $0,49 \mathrm{aA}$ & $0,45 \mathrm{aA}$ & $0,53 \mathrm{aA}$ & & & & \\
\hline & $20-30$ & $0,24 \mathrm{bB}$ & $0,29 \mathrm{bAB}$ & $0,28 \mathrm{bAB}$ & $0,37 \mathrm{bA}$ & & & & \\
\hline & $30-40$ & $0,22 \mathrm{bB}$ & $0,30 \mathrm{bAB}$ & $0,32 \mathrm{bAB}$ & $0,32 \mathrm{bA}$ & & & & \\
\hline \multirow{5}{*}{ m $(\%)$} & $\mathrm{cm}$ & & & & & & & & \\
\hline & $0-10$ & $21,12 \mathrm{bA}$ & $14,38 \mathrm{bA}$ & $13,74 \mathrm{cA}$ & $10,74 \mathrm{bA}$ & \multirow{4}{*}{18,24} & \multirow{4}{*}{5,64} & \multirow{4}{*}{5,64} & \multirow{4}{*}{11,28} \\
\hline & $10-20$ & $33,92 \mathrm{aC}$ & $42,05 \mathrm{aBC}$ & $49,24 \mathrm{abAB}$ & $56,48 \mathrm{aA}$ & & & & \\
\hline & $20-30$ & $34,26 \mathrm{aB}$ & $38,93 \mathrm{aAB}$ & 39,18 bAB & $49,49 \mathrm{aA}$ & & & & \\
\hline & $30-40$ & $40,29 \mathrm{aB}$ & $45,05 \mathrm{aB}$ & $56,86 \mathrm{aA}$ & $47,11 \mathrm{aAB}$ & & & & \\
\hline \multirow{5}{*}{$\mathrm{P}\left(\mathrm{mg} \mathrm{dm}^{-3}\right)$} & $\mathrm{cm}$ & & & & & \multirow{5}{*}{7,48} & \multirow{5}{*}{0,04} & \multirow{5}{*}{0,04} & \\
\hline & $0-10$ & $0,94 \mathrm{aAB}$ & $0,97 \mathrm{aA}$ & $0,87 \mathrm{aBC}$ & $0,82 \mathrm{aC}$ & & & & \\
\hline & $10-20$ & $0,77 \mathrm{bA}$ & $0,75 \mathrm{bA}$ & $0,70 \mathrm{bA}$ & $0,60 \mathrm{bB}$ & & & & $0 \cap 8$ \\
\hline & $20-30$ & $0,59 \mathrm{cA}$ & $0,55 \mathrm{cA}$ & $0,57 \mathrm{cA}$ & $0,57 \mathrm{bA}$ & & & & 0,00 \\
\hline & $30-40$ & $0,63 \mathrm{cA}$ & $0,63 \mathrm{cA}$ & $0,57 \mathrm{cA}$ & $0,55 \mathrm{bA}$ & & & & \\
\hline & $\mathrm{cm}$ & & & & & & & & \\
\hline & $0-10$ & $27,55 \mathrm{bA}$ & $22,48 \mathrm{bB}$ & $19,49 \mathrm{abB}$ & $27,77 \mathrm{bA}$ & & & & \\
\hline M.O. $\left(\mathrm{g} \mathrm{dm}^{-3}\right)$ & $10-20$ & $36,92 \mathrm{aA}$ & $24,49 \mathrm{bC}$ & $14,00 \mathrm{cD}$ & $29,95 \mathrm{abB}$ & 777 & 160 & 160 & 221 \\
\hline & $20-30$ & $34,30 \mathrm{aA}$ & $28,05 \mathrm{aB}$ & $20,42 \mathrm{aC}$ & $31,93 \mathrm{aA}$ & 1,11 & 1,00 & 1,00 & 3,21 \\
\hline & $30-40$ & $19,02 \mathrm{cB}$ & $22,75 \mathrm{bA}$ & $16,76 \mathrm{bcB}$ & $19,36 \mathrm{cB}$ & & & & \\
\hline & $\mathrm{cm}$ & & & & & & & & \\
\hline & $0-10$ & $1,23 \mathrm{aB}$ & $1,29 \mathrm{aA}$ & $1,19 \mathrm{aC}$ & $1,17 \mathrm{aC}$ & & & & \\
\hline $\mathrm{K}^{+}\left(\mathrm{mg} \mathrm{dm}^{-3}\right)$ & $10-20$ & $1,05 \mathrm{cB}$ & $1,08 \mathrm{bA}$ & $1,05 \mathrm{bcB}$ & $0,98 \mathrm{cC}$ & 123 & 011 & 011 & 023 \\
\hline & $20-30$ & $1,07 \mathrm{bcA}$ & $1,08 \mathrm{bA}$ & $1,07 \mathrm{bA}$ & $1,02 \mathrm{bcB}$ & 1,23 & 0,11 & 0,11 & 0,23 \\
\hline & $30-40$ & $1,07 \mathrm{bA}$ & $1,07 \mathrm{bAB}$ & $1,05 \mathrm{cBC}$ & $1,04 \mathrm{bC}$ & & & & \\
\hline & $\mathrm{cm}$ & & & & & & & & \\
\hline & $0-10$ & $0,92 \mathrm{aB}$ & $0,99 \mathrm{aA}$ & $0,78 \mathrm{aC}$ & $0,75 \mathrm{aC}$ & & & & \\
\hline $\mathrm{Ca}^{2+}\left(\mathrm{cmol}_{\mathrm{c}} \mathrm{dm}^{-3}\right)$ & $10-20$ & $0,70 \mathrm{bA}$ & $0,42 \mathrm{bB}$ & $0,31 \mathrm{bC}$ & $0,23 \mathrm{bD}$ & 004 & 003 & 003 & $0 \Omega 6$ \\
\hline & $20-30$ & $0,22 \mathrm{cA}$ & $0,22 \mathrm{cA}$ & $0,23 \mathrm{cA}$ & $0,18 \mathrm{bA}$ & 9,04 & 0,03 & 0,03 & 0,00 \\
\hline & $30-40$ & $0,23 \mathrm{cA}$ & $0,23 \mathrm{cA}$ & $0,18 \mathrm{cAB}$ & $0,12 \mathrm{cB}$ & & & & \\
\hline
\end{tabular}


continuação

\begin{tabular}{|c|c|c|c|c|c|c|c|c|c|}
\hline \multirow{5}{*}{$\mathrm{Mg}^{2+}\left(\mathrm{cmol}_{\mathrm{c}} \mathrm{dm}^{-3}\right)$} & \multicolumn{5}{|l|}{$\mathrm{cm}$} & \multirow{5}{*}{23,08} & \multirow{5}{*}{0,05} & \multirow{5}{*}{0,05} & \multirow{5}{*}{0,10} \\
\hline & $0-10$ & $0,68 \mathrm{aA}$ & $0,56 \mathrm{aB}$ & $0,38 \mathrm{aC}$ & $0,66 \mathrm{aAB}$ & & & & \\
\hline & $10-20$ & $0,15 \mathrm{bcA}$ & $0,23 \mathrm{bA}$ & $0,14 \mathrm{bA}$ & $0,15 \mathrm{bA}$ & & & & \\
\hline & $20-30$ & $0,21 \mathrm{bA}$ & $0,22 \mathrm{bA}$ & $0,18 \mathrm{bA}$ & $0,18 \mathrm{bA}$ & & & & \\
\hline & $30-40$ & $0,07 \mathrm{cB}$ & $0,11 \mathrm{cB}$ & $0,03 \mathrm{cB}$ & $0,22 \mathrm{bA}$ & & & & \\
\hline & $\mathrm{cm}$ & & & & & & & & \\
\hline & $0-10$ & $1,64 \mathrm{aA}$ & $1,58 \mathrm{aA}$ & $1,19 \mathrm{aC}$ & $1,44 \mathrm{aB}$ & & & & \\
\hline $\mathrm{SB}\left(\mathrm{cmol}_{\mathrm{c}} \mathrm{dm}^{-3}\right)$ & $10-20$ & $0,87 \mathrm{bA}$ & $0,67 \mathrm{bB}$ & $0,47 \mathrm{bC}$ & $0,41 \mathrm{bC}$ & 1160 & 007 & 007 & 0.14 \\
\hline & $20-30$ & $0,45 \mathrm{cA}$ & $0,46 \mathrm{cA}$ & $0,43 \mathrm{bA}$ & $0,38 \mathrm{bA}$ & 11,00 & 0,07 & 0,07 & 0,14 \\
\hline & $30-40$ & $0,33 \mathrm{cA}$ & $0,36 \mathrm{cA}$ & $0,23 \mathrm{cA}$ & $0,37 \mathrm{bA}$ & & & & \\
\hline & $\mathrm{cm}$ & & & & & & & & \\
\hline & $0-10$ & $2,07 \mathrm{aA}$ & $1,85 \mathrm{aB}$ & $1,38 \mathrm{aD}$ & $1,61 \mathrm{aC}$ & & & & \\
\hline $\mathrm{CTC}_{\mathrm{e}}\left(\mathrm{cmol}_{\mathrm{c}} \mathrm{dm}^{-3}\right)$ & $10-20$ & $1,32 \mathrm{bA}$ & $1,16 \mathrm{bB}$ & $0,93 \mathrm{bC}$ & $0,94 \mathrm{bC}$ & 887 & 008 & 008 & 015 \\
\hline & $20-30$ & $0,69 \mathrm{cA}$ & $0,75 \mathrm{cA}$ & $0,71 \mathrm{cA}$ & $0,75 \mathrm{cA}$ & 8,81 & 0,08 & 0,08 & 0,15 \\
\hline & $30-40$ & $0,55 \mathrm{cA}$ & $0,66 \mathrm{cA}$ & $0,55 \mathrm{dA}$ & $0,69 \mathrm{cA}$ & & & & \\
\hline & $\mathrm{cm}$ & & & & & & & & \\
\hline & $0-10$ & $3,67 \mathrm{aB}$ & $3,97 \mathrm{aA}$ & $2,51 \mathrm{aD}$ & $3,10 \mathrm{aC}$ & & & & \\
\hline $\operatorname{CTC}\left(\mathrm{cmol}_{\mathrm{c}} \mathrm{dm}^{-3}\right)$ & $10-20$ & $2,02 \mathrm{bB}$ & $2,59 \mathrm{bA}$ & $2,14 \mathrm{bB}$ & $1,98 \mathrm{bB}$ & 616 & 1 & 0 & 2 \\
\hline & $20-30$ & $1,20 \mathrm{cD}$ & $2,06 \mathrm{cA}$ & $1,56 \mathrm{cC}$ & $1,81 \mathrm{bB}$ & 0,40 & 0,1 & 0,1 & 0,23 \\
\hline & $30-40$ & $1,19 \mathrm{cA}$ & $1,37 \mathrm{~dB}$ & $1,40 \mathrm{cB}$ & $1,75 \mathrm{cA}$ & & & & \\
\hline & $\mathrm{cm}$ & & & & & & & & \\
\hline & $0-10$ & $44,70 \mathrm{aAB}$ & $39,89 \mathrm{aB}$ & $47,43 \mathrm{aA}$ & $46,25 \mathrm{aA}$ & & & & \\
\hline V (\%) & $10-20$ & $43,23 \mathrm{abA}$ & $25,88 \mathrm{bB}$ & $22,00 \mathrm{bcB}$ & $20,62 \mathrm{bB}$ & 1190 & 284 & 284 & 569 \\
\hline & $20-30$ & $37,84 \mathrm{bA}$ & $22,36 \mathrm{bBC}$ & $27,62 \mathrm{bB}$ & $21,29 \mathrm{bC}$ & 11,90 & & 2,04 & \\
\hline & $30-40$ & $27,90 \mathrm{cA}$ & $26,59 \mathrm{bA}$ & $16,55 \mathrm{cB}$ & $20,91 \mathrm{bB}$ & & & & \\
\hline
\end{tabular}

Médias seguidas por letras iguais, minúsculas na coluna e maiúscula na linha, não se diferem entre si pelo teste de Tukey ao nível de $5 \%$ de probabilidade. DMS = diferença mínima significativa. Prof. = profundidade. Inter. $=$ interação (dose $\mathrm{x}$ prof.).

Fonte: Elaboração dos autores.

Já a redução do $\mathrm{pH}$ nas doses maiores, possivelmente, ocorreu devido à nitrificação ou oxidação do $\mathrm{N}$ amoniacal a nitrato, pois nesse processo há formação de dois prótons $\left(\mathrm{H}^{+}\right)$para cada íon de $\mathrm{NH}_{4}^{+}$nitrificado (SOUSA; MIRANDA; OLIVEIRA, 2007). Além disso, a própria absorção do amônio pelas plantas promove a redução do $\mathrm{pH}$ do solo devido à extrusão de prótons da raiz para a rizosfera (MARSCHNER, 1995). Nesse sentido as altas concentrações de $\mathrm{N}$ amoniacal no RLF (Tabela 1) reduziu o $\mathrm{pH}$.

Pires et al. (2008) verificaram que farinha de ossos e carne também reduziram o pH do solo. Já Costa et al. (2008) adubando capim-Marandu com uréia e sulfato de amônio e Primavesi et al. (2006) fertilizando capim-Coastcross com uréia e nitrato de amônio encontraram redução no $\mathrm{pH}$ do solo. Nas camadas 10-20, 20-30 e 30-40 cm a maior dose de RLF proporcionou os menores valores de $\mathrm{pH}$. Assim, as reduções do $\mathrm{pH}$ devido ao aumento das doses não ficaram restritas a camada superficial. Esses resultados podem ser atribuídos à própria característica física do resíduo e as intrínsecas do solo que podem ter lixiviado o $\mathrm{N}$ amoniacal para essas camadas e intensificado o processo de nitrificação. Em profundidade, observou-se que em todos os tratamentos o valor de $\mathrm{pH}$ foi maior na camada 0-10 cm, não havendo modificações nas demais camadas.

Na camada de 0-10 $\mathrm{cm}$ a dose de $37,5 \mathrm{~m}^{3} \mathrm{ha}^{-1}$ proporcionou o maior valor de acidez potencial $(\mathrm{H}+\mathrm{Al})$ seguido da testemunha e das doses de 112,5 
e $75 \mathrm{~m}^{3} \mathrm{ha}^{-1}$, respectivamente (Tabela 3 ). Na camada 10-20 $\mathrm{cm}$ a dose $37,5 \mathrm{~m}^{3}$ ha $^{-1}$ manteve valores superiores aos demais tratamentos. O segundo maior valor foi observado nas doses de 75 e $112,5 \mathrm{~m}^{3} \mathrm{ha}^{-1}$ e o menor na testemunha. Já nas profundidades de 20-30 e 30-40 cm as doses de 37,5; 75 e 112,5 $\mathrm{m}^{3}$ ha $^{-1}$ apresentaram maior valor de $\mathrm{H}+\mathrm{Al} \mathrm{em}$ relação à testemunha. Observou-se em todos os tratamentos que o valor de $\mathrm{H}+\mathrm{Al}$ foi alterado em função da profundidade e que os maiores valores foram encontrados na profundidade de $0-10 \mathrm{~cm}$ com aplicação de 0; 37,5 e 112,5 $\mathrm{m}^{3}$ ha ${ }^{-1}$ de RLF.

Em função da profundidade foi possível verificar que o teor de $\mathrm{Al}^{3+}$ foi maior na camada de 10-20 $\mathrm{cm}$ em todos os tratamentos (Tabela 3 ). Na camada 0-10 $\mathrm{cm}$ as doses de 112,5 e $75 \mathrm{~m}^{3} \mathrm{ha}^{-1}$ apresentaram valores menores de $\mathrm{Al}^{3+}$. Essas reduções nos teores de $\mathrm{Al}^{3+}$ trocável também foram observadas por Ceretta et al. (2003) com a aplicação de esterco líquido de suínos em pastagem natural. No entanto, Roscoe et al. (2006) trabalhando com resíduo sólido de frigorífico como fertilizante observaram que as maiores aplicações desse composto orgânico aumentaram os teores de $\mathrm{Al}^{3+}$.

A redução do $\mathrm{Al}^{3+}$ com aplicação de RLF devese à provável a complexação do alumínio pelos ácidos orgânicos (húmicos e fúlvicos) adicionados ao solo via resíduo líquido. A complexação do $\mathrm{Al}^{3+}$ pelos compostos orgânicos foi reportada por Pavan (1983); Sidiras e Pavan (1985); Miyazawa, Chierice e Pavan (1992) e Theodoro et al. (2003). Nas profundidades de $20-30$ e $30-40 \mathrm{~cm}$ a dose de $112,5 \mathrm{~m}^{3} \mathrm{~h}^{-1}$ teor de $\mathrm{Al}^{3+}$ foi maior o que indica que a complexação deste pelos ácidos orgânicos, ficou restrita a camada de $0-10 \mathrm{~cm}$ de profundidade.

A saturação por $\mathrm{Al}^{3+}(\mathrm{m} \%)$ (Tabela 3$)$ não foi afetada pelos tratamentos na camada de $0-10 \mathrm{~cm}$. Nas camadas do solo de $10-20$ e $20-30 \mathrm{~cm}$ a dose de $112,5 \mathrm{~m}^{3} \mathrm{ha}^{-1}$ teve maior saturação que à testemunha. Já na profundidade de $30-40 \mathrm{~cm}$ os resultados promovidos pela dose de $75 \mathrm{~m}^{3} \mathrm{ha}^{-1}$ foi superior à testemunha. Scherer, Baldisser e Nesi (2007) utilizando esterco de suínos obtiveram respostas semelhantes. A m\% modificou-se em profundidade com maiores valores em todos os tratamentos, nas camadas de 10 a $40 \mathrm{~cm}$.

As doses crescentes de RLF aplicadas ao solo em estudo ocasionaram alterações nas concentrações de P (Tabela 3) disponível no solo somente nas camadas de 0-10 e 10-20 cm. Em função da profundidade todos os tratamentos apresentaram maior concentração de $\mathrm{P}$ na profundidade de 0-10 cm e menores valores nas camadas de $20-30$ e 30 40 para os tratamentos $0 ; 37,5$ e $75 \mathrm{~m}^{3} \mathrm{ha}^{-1}$ e nas profundidades de 10 à $40 \mathrm{~cm}$ para o tratamento de $112,5 \mathrm{~m}^{3} \mathrm{ha}^{-1}$. Na profundidade $0-10 \mathrm{~cm}$ a maior disponibilidade foi encontrada na testemunha e na dose de $37,5 \mathrm{~m}^{3} \mathrm{ha}^{-1}$ os quais não se diferiram. Este resultado pode ser explicado pela baixa produção do capim-Marandu que exportou menor quantidade desse nutriente nas doses menores (Tabela 4). As doses de 75 e $112,5 \mathrm{~m}^{3} \mathrm{ha}^{-1}$ de RLF apresentaram concentrações inferiores à testemunha e à dose de $37,5 \mathrm{~m}^{3} \mathrm{ha}^{-1}$, no entanto essas doses responderam por produção de MS superiores com o aumento das doses de RLF. O que também aumentou linearmente a concentração de $\mathrm{P}$ e dos nutrientes $\mathrm{N}$ e K na MS do capim-marandu.

Na camada $10-20 \mathrm{~cm}$ o baixo teor de P no solo que recebeu a maior aplicação de RLF permaneceu em relação à testemunha e as doses de 37,5 e $75 \mathrm{~m}^{3}$ $\mathrm{ha}^{-1}$ os quais não se. Isso pode ter ocorrido devido ao desenvolvido do sistema radicular das plantas, pois o crescimento das forrageiras não se restringe somente a parte aérea, o que possivelmente favoreceu a exploração do solo e resultou em maiores extrações de P pela cultura. Essa observação é evidenciada por Monteiro et al. (1995) que cultivando capimMarandu em solução nutritiva completa verificaram aumentos nas produções de MS radicular e da parte aérea concomitantemente, em relação ao tratamento com omissão de P. Nesse mesmo sentido, Santos Júnior (2001) obteve incremento na produção de MS radicular de capim-Marandu com o aumento das doses de $\mathrm{N}$ sob condições de 
casa-de-vegetação. Assim sendo, o possível aporte de maiores quantidades de $\mathrm{N}$ amoniacal na dose de $112,5 \mathrm{~m}^{3} \mathrm{ha}^{-1}$ tenha favorecido o crescimento do sistema radicular.

Os teores de matéria orgânica (M.O.) do solo (Tabela 3) não apresentaram incrementos significativos com as aplicações de RLF sendo que a testemunha apresentou valores superiores aos demais na profundidade de $10-20 \mathrm{~cm}$. Nas camadas de $0-10$ e $10-20 \mathrm{~cm}$, a testemunha e a dose de $112,5 \mathrm{~m}^{3} \mathrm{ha}^{-1}$ de $\mathrm{RLF}$ proporcionaram os maiores incrementos. Oliveira et al. (2005) trabalhando com a fertilização de $\mathrm{N}$ e $\mathrm{S}$ por dois anos na recuperação de pastagem de capim-Marandu, em Neossolo Quartzarênico, observaram reduções no teor de M.O. do solo em função do tempo. Já Batista (2006), estudando doses combinadas de N e S na implantação do capim-Marandu em substituição ao capim-braquiária em degradação e em solo com baixa M.O., observou que o $\mathrm{N}$ aplicado em altas doses acelera a decomposição da M.O., promovendo a redução do seu teor.

Tabela 4. Produção de matéria seca total (MST) e concentração de nitrogênio $(\mathrm{N})$, fósforo $(\mathrm{P})$ e potássio $(\mathrm{K})$ na fitomassa da Brachiaria brizantha cv. Marandu após receber doses de resíduo líquido de frigorífico (RLF).

\begin{tabular}{ccccc}
\hline Dose de RLF & MST $^{(1)}$ & \multicolumn{3}{c}{ Nutrientes } \\
\hline \multirow{2}{*}{$\mathrm{m}^{3} \mathrm{ha}^{-1}$} & \multirow{2}{*}{$\mathrm{kg} \mathrm{ha}^{-1}$} & $\mathrm{~N}$ & $\mathrm{P}$ & $\mathrm{kg} \mathrm{ha}$ \\
\cline { 3 - 5 } & & \multicolumn{3}{c}{$\mathrm{kg}^{-1}$} \\
37,00 & 755,54 & 10,98 & 0,33 & 0,14 \\
75,00 & $2.665,52$ & 42,84 & 1,36 & 0,17 \\
112,50 & $3.113,76$ & 51,65 & 1,66 & 0,18 \\
\hline $\mathrm{CV}(\%)$ & $3.878,24$ & 67,24 & 2,37 & 0,20 \\
Equação & 5,92 & 10,29 & 12,48 & 9,88 \\
$\mathrm{R}^{2}$ & $\hat{\mathrm{Y}}=1.130,80+26,17^{*} \mathrm{X}$ & $\hat{\mathrm{Y}}=16,08+0,47^{*} \mathrm{X}$ & $\hat{\mathrm{Y}}=0,46+0,017^{*} \mathrm{X}$ & $\hat{\mathrm{Y}}=0,15+0,0004^{*} \mathrm{X}$ \\
\hline
\end{tabular}

${ }^{(1)}$ MST: produção de matéria seca total de dois cortes sucessivos. *: significativo a 5\% de probabilidade pelo teste $\mathrm{F}$. Fonte: Elaboração dos autores.

De acordo com Plaza et al. (2005) e Carter e Campbell (2006) a aplicação de estercos líquidos nem sempre aumenta o teor de C orgânico, pois a M.O. desses resíduos orgânicos é composta em grande parte por C orgânico rapidamente decomposto que não contribui significativamente para a M.O. estável do solo. Além disso, o alto teor de $\mathrm{C}$ lábil e a disponibilidade de $\mathrm{N}$ e $\mathrm{P}$ podem acelerar a decomposição de resíduos vegetais e do C já presente no solo (ROCHETTE; ANGERS; CÔTÉ, 2000; CHANTIGNY; ROCHETTE; ANGERS, 2001; PLAZA et al., 2005). Assim, as altas concentrações de N no RLF, possivelmente, favoreceram a decomposição da M.O. do solo em estudo.
As concentrações de $\mathrm{K}^{+}, \mathrm{Ca}^{2+}$ e $\mathrm{Mg}^{2+}$ foram influenciadas pela profundidade com maiores valores na profundidade de $0-10 \mathrm{~cm}$ e reduziram com o aumento da profundidade em todos os tratamentos estudados (Tabela 3 ).

As doses crescentes de RLF alteraram as concentrações de $\mathrm{Ca}^{2+}$ no solo, exceto na camada de 20-30 onde as doses de RLF não se diferiram. Na profundidade de $0-10 \mathrm{~cm}$ os maiores teores de $\mathrm{Ca}^{2+}$ foram observados na dose de $37,5 \mathrm{~m}^{3} \mathrm{ha}^{-1}$ de $\mathrm{RLF}$ e na testemunha, respectivamente. $\mathrm{O}$ menor aporte de nutrientes reduziu excessivamente o desenvolvimento e produção de MS da forrageira (Tabela 4), que provavelmente reduziu o acumulo e exportação de $\mathrm{Ca}^{2+}$. A correção do solo com calcário 
e a aplicação de $37,5 \mathrm{~m}^{3}$ ha ${ }^{-1}$ de RLF contribuíram para a manutenção desse nutriente no solo, pois as concentrações de $\mathrm{Ca}^{2+}$ neste tratamento são superiores aos demais e aos teores observados no momento da implantação do experimento (Tabela 1). Nesse sentido, em sistemas de alta produtividade de forragem onde a exportação de nutrientes pela cultura é alta, os incrementos de $\mathrm{Ca}^{2+}$ nas maiores doses de RLF, não foram suficientes para aumentar as concentrações deste no solo. Fonseca (2005) também obteve respostas similares quando irrigou capim Tifton 85 com efluente secundário de estação de tratamento de esgoto.

A concentração de $\mathrm{Ca}^{2+}$ na camada 10-20 $\mathrm{cm}$ reduziu com aumento das doses de RLF, o que está relacionado diretamente com o exposto anteriormente. Segundo Werner, Paulino e Cantarella (1996) a quantidade de nutrientes extraídos do solo pela planta forrageira será maior quanto maior for a produção da pastagem. Assim sendo, a testemunha obteve maior teor de $\mathrm{Ca}^{2+}$ no solo que a dose de 37,5 $\mathrm{m}^{3} \mathrm{ha}^{-1}$ que foi superior a de $75 \mathrm{~m}^{3} \mathrm{ha}^{-1}$ e por final, teve concentração superior à aplicação de $112,5 \mathrm{~m}^{3}$ ha $^{-1}$.

As concentrações de $\mathrm{Mg}^{2+}$ (Tabela 3) foram influenciadas pelos RLF somente nas profundidades de 0-10 e 30-40 cm, nas demais camadas os tratamentos não se diferiram. $\mathrm{Na}$ camada de 0-10 $\mathrm{cm}$ a testemunha apresentou concentrações de $\mathrm{Mg}^{2+}$ maiores que as doses de 37,5 e $75 \mathrm{~m}^{3} \mathrm{ha}^{-1}$ de RLF, não se diferindo da dose de $112,5 \mathrm{~m}^{3} \mathrm{ha}^{-1}$ a qual não se diferiu da dose de $37,5 \mathrm{~m}^{3} \mathrm{ha}^{-1}$. Assim, a aplicação de $112,5 \mathrm{~m}^{3} \mathrm{ha}^{-1}$ de RLF em pastagens de capim-Marandu, aumentou a concentração de $\mathrm{Mg}^{2+}$ trocável, que apesar do aumento na produção de MS de forragem e provável exportação deste nutriente, manteve os teores iguais aos tratamentos que praticamente não tiveram produtividade. Já a dose intermediária $\left(75 \mathrm{~m}^{3} \mathrm{ha}^{-1}\right)$ não foi capaz de aliar boa produção de gramínea com manutenção de $\mathrm{Mg}^{2+}$ no solo, fazendo assim com que este tratamento tenha apresentado menor concentração deste nutriente no solo em relação aos demais tratamentos. Na profundidade de $30-40 \mathrm{~cm}$ a dose de $112,5 \mathrm{~m}^{3} \mathrm{ha}^{-1}$ de RLF foi superior quanto à concentração de $\mathrm{Mg}^{2+}$ em comparação aos outros tratamentos.

A comparação dos tratamentos na camada 0-10 $\mathrm{cm}$ mostra que a dose de $37,5 \mathrm{~m}^{3} \mathrm{ha}^{-1}$ apresenta maiores teores de $\mathrm{K}^{+}$em relação à testemunha $\mathrm{e}$ as doses 75 e $112,5 \mathrm{~m}^{3} \mathrm{ha}^{-1}$ sendo que as maiores aplicações de RLF não se diferirem (Tabela 3). Na profundidade de $10-20 \mathrm{~cm}$ a concentração de $\mathrm{K}^{+}$na dose de $37,5 \mathrm{~m}^{3}$ ha-1 se manteve superior em relação à testemunha e as maiores doses de RLF, a aplicação de $75 \mathrm{~m}^{3}$ ha $^{-1}$ não se diferiu estatisticamente da testemunha. Ceretta et al. (2003) encontrou reduções nos teores de $\mathrm{K}^{+}$disponível no solo com a aplicação de esterco líquido de suínos em pastagem natural até a profundidade de $10-20 \mathrm{~cm}$. O tratamento de $112,5 \mathrm{~m}^{3} \mathrm{ha}^{-1}$ apresentou menores teores de $\mathrm{K}^{+}$na camada de 20-30 cm em relação aos demais, esses não se diferiram estatisticamente. Esses resultados

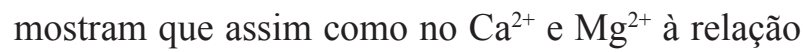
com a produção de MS, demanda e exportação de nutrientes (Tabela 4) nessas condições. Entretanto os teores de $\mathrm{K}^{+}$no tecido vegetal estavam em patamares abaixo dos recomendados pela literatura (WERNER; PAULINO; CANTARELLA, 1996) o que indica que nenhum tratamento forneceu níveis adequados deste nutriente à cultura.

Os valores de $\mathrm{SB}, \mathrm{CTC}_{\text {efetiva }}, \mathrm{CTC}_{\mathrm{pH} 7}$ e V\% foram influenciados pela profundidade com maiores na camada de $0-10 \mathrm{~cm}$ de profundidade em todos os tratamentos (Tabela 3). Verificou-se que houve diferença significativa apenas nas camadas 0-10 e 10-20 cm para a soma de bases (SB). O uso de RLF no solo reduziu o valor da $\mathrm{SB}$ nas doses de 112,5 e $75 \mathrm{~m}^{3} \mathrm{ha}^{-1}$, respectivamente, sendo que essas se diferiram na camada de $10-20 \mathrm{~cm}$, uma vez que os teores de $\mathrm{Ca}^{2+}, \mathrm{Mg}^{2+}$ e $\mathrm{K}^{+}$nos tratamentos com aplicação de maiores doses de RLF foram inferiores a testemunha e a dose de $37,5 \mathrm{~m}^{3}$ ha $^{-1}$ devido à possível exportação desses nutrientes pelo capimMarandu com a aplicação de 75 e $112,5 \mathrm{~m}^{3} \mathrm{ha}^{-1}$ de RLF. Observa-se também, que os tratamentos que apresentaram maiores $\mathrm{SB}$, foram os que tiveram 
altos teores de $\mathrm{P}$ e $\mathrm{pH}$, no entanto essa contribuição significativa para a melhoria das características químicas do solo não refletiu em maiores produções de MS da forragem.

Os maiores valores da capacidade de troca de cátions a $\mathrm{pH} 7\left(\mathrm{CTC}_{\mathrm{pH} 7}\right)$ do solo (Tabela 3$)$ foram encontrados na dose de $37,5 \mathrm{~m}^{3} \mathrm{ha}^{-1}$ na camada de 0-10 cm, em seguida a testemunha e posteriormente os tratamentos 112,5 e $75 \mathrm{~m}^{3} \mathrm{ha}^{-1}$. O uso de $37,5 \mathrm{~m}^{3}$ ha $^{-1}$ de RLF aumentou a $\mathrm{CTC}_{\mathrm{pH} 7}$ na profundidade de 10-20 cm, os demais tratamentos não se diferiram. Esses resultados é reflexo direto de maiores SB e da $\mathrm{H}+\mathrm{Al}$ desse tratamento. Na camada de 20-30 $\mathrm{cm}$ esse comportamento se manteve com maiores valores de CTC ${ }_{\mathrm{pH} 7}$ no tratamento $37,5 \mathrm{~m}^{3} \mathrm{ha}^{-1}$ seguido pelas doses de 75 e $112,5 \mathrm{~m}^{3} \mathrm{ha}^{-1}$ com menor valor na testemunha.

Nessas condições, observou-se resposta significativa ao uso do RLF, com maior porcentagem de saturação de bases (V\%) (Tabela 3) nas doses de 112,5 e $75 \mathrm{~m}^{3} \mathrm{ha}^{-1}$ e menores na aplicação de $37,5 \mathrm{~m}^{3} \mathrm{ha}^{-1}$ e na testemunha na profundidade de 0-10 cm. Nas camadas de $10-20$ e $20-30 \mathrm{~cm}$ a aplicação de doses crescentes de RLF reduziu a V\%, esses tratamentos não se diferiram. Já na profundidade de $30-40 \mathrm{~cm}$ a dose de $37,5 \mathrm{~m}^{3} \mathrm{ha}^{-1}$ e a testemunha tiveram os maiores valores de V\%. Moreti et al. (2007) verificaram que a adubação, de um Latossolo Vermelho distrófico, com esterco de galinha aumentou os valores de $\mathrm{SB}$ e a $\mathrm{CTC}_{\mathrm{pH}}$.

Os maiores teores de bases trocáveis $\left(\mathrm{Ca}^{2+}\right.$, $\mathrm{Mg}^{2+}, \mathrm{K}^{+}$) e dos teores de $\mathrm{Al}^{3+}$ na testemunha e na dose de $37,5 \mathrm{~m}^{3} \mathrm{ha}^{-1}$ de RLF, proporcionaram valores também, maiores na capacidade efetiva de troca de cátions $\left(\mathrm{CTC}_{\text {efetiva }}\right)$ do solo (Tabela 3 ) nas camadas 0-10 e 10-20 cm, as doses de 75 e 112,5 $\mathrm{m}^{3}$ ha $^{-1}$ não se diferiram. Nas profundidades 2030 e 30-40 cm não se observou diferença entre os tratamentos estudados. Observa-se que a provável extração de bases pelas maiores doses empregadas resulta em valores menores da $\mathrm{CTC}_{\text {efetiva }}$. Roscoe et al. (2006) observaram tendência de redução gradual da $\mathrm{CTC}_{\text {efetiva }}$ do solo com a aplicação de fertilizante mineral ao solo tendo como causa uma provável

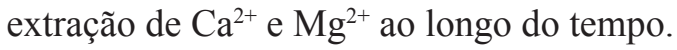

\section{Conclusões}

A adubação orgânica em Neossolo Quartzarênico por meio da aplicação de 75 e $112,5 \mathrm{~m}^{3} \mathrm{ha}^{-1}$ de RLF reduziu o $\mathrm{pH}, \mathrm{P}, \mathrm{Ca}, \mathrm{K}, \mathrm{SB}, \mathrm{CTC}_{\mathrm{e}}$ e $\mathrm{CTC}_{\mathrm{pH} 7,0} \mathrm{e} \mathrm{Al}^{3+} \mathrm{e}$ aumentou a $\mathrm{V}$, na camada de 0-10.

Os teores de matéria orgânica do solo não aumentam com a aplicação de doses crescentes de RLF sob pastagem de capim-Marandu.

$\mathrm{O} \mathrm{pH}$, soma de bases, CTC efetiva, CTC ${ }_{\mathrm{pH}}$, saturação por bases, $\mathrm{K}^{+}, \mathrm{Ca}^{2+} \mathrm{e} \mathrm{Mg}^{2+}$ são influenciados pela profundidade com maiores valores na camada de $0-10 \mathrm{~cm}$ de profundidade em relação às demais, em todos os tratamentos. Reformular!!

\section{Agradecimentos}

Ao CNPq pelo apoio com as Bolsas no País/ Iniciação Científica-Edital MCT/CNPq n. ${ }^{\circ}$ 12/2010 - IC, b) Edital MCT/CNPq 10/2010 - Apoio Técnico/Edital MCT/CNPq 10/2010 - AT- NS (Nível Superior); e c) Bolsas no País/Produtividade em Pesquisa - PQ - 2009 - Orientador (COAGR/ CGAPB/DABS).

\section{Referências}

ABREU JUNIOR, C. H.; BOARETTO, A. E.; MURAOKA, T.; KIEHL, J. C. Uso agrícola de resíduos orgânicos potencialmente poluentes: propriedades químicas do solo e produção vegetal. In: TORRADO, P. V.; ALLEONI, L. R. F.; COOPER, M.; SILVA, A. P.; CARDOSO, E. J. (Ed.). Tópicos em ciência do solo. Viçosa, MG: Sociedade Brasileira de Ciência do Solo, 2005. v. 4. p. 391-470.

AMERICAN PUBLIC HEALTH ASSOCIATION APHA. Standard methods for the examination of water and wastewater. 19. ed. Washington, D. C., 1995. $1155 \mathrm{p}$.

BATISTA, K. Nitrogênio e enxofre na implantação do capim-marandu em substituição ao capim-braquiária em 
degradação num solo com baixa matéria orgânica. 2006. Tese (Doutorado em Agronomia) - Escola Superior de Agricultura Luiz de Queiroz. Universidade de São Paulo, Piracicaba.

CARTER, M. R.; CAMPBELL, A. J. Influence of tillage and liquid swine manure on productivity of a soybeanbarley rotation and some properties of a fine sandy loam in Prince Edward Island. Canadian Journal of Soil Science, Ottawa, v. 86, n. 4, p. 741-748, 2006.

CERETTA, C. A.; DURIGON, R.; BASSO, C. J.; BARCELLOS, L. A. R.; VIEIRA, F. C. B. Características químicas de solo sob aplicação de esterco líquido de suínos em pastagem natural. Pesquisa Agropecuária Brasileira, Brasília, v. 38, n. 6, p. 729-735, 2003.

CHANTIGNY, M. H.; ROCHETTE, P.; ANGERS, D. A. Short-term $\mathrm{C}$ and $\mathrm{N}$ dynamics in a soil amended with pig slurry and barley straw: a field experiment. Canadian Journal of Soil Science, Ottawa, v. 81, n. 2, p. 131-137, 2001.

ClAessen, M. E. C. (Org.). Manual de métodos de análise de solo. $2^{\text {nd }} \mathrm{ed}$. Rio de Janeiro: Embrapa-CNPS, 1997. 212 p. (Embrapa CNPS. Documentos, 1).

COSTA, K. A. P.; FAQUIN, V.; OLIVEIRA, I. P.; RODRIGUES, C.; SEVERIANO, E. C. Doses e fontes de nitrogênio em pastagem de capim-marandu. I alterações nas características químicas do solo. Revista Brasileira de Ciência do Solo, Viçosa, MG, v. 32, n. 4, p. 591-1599, 2008.

EMPRESA BRASILEIRA DE PESQUISA AGROPECUÁRIA - EMBRAPA. Centro Nacional de Pesquisa de Solos (Rio de Janeiro, RJ). Sistema brasileiro de classificação de solos. Brasília: EmbrapaSPI, Embrapa-CNPS, 1999. 412 p.

FONSECA, A. F. Viabilidade agronômica-ambiental da disposição de efluente de esgoto tratado em um sistema solo-planta. 2005. Tese (Doutorado em Agronomia) - Escola Superior de Agricultura Luiz de Queiroz. Universidade de São Paulo, Piracicaba.

GASI, T. M. T. Caracterização, reaproveitamento e tratamento de resíduos de frigorificos, abatedouros e graxarias. São Paulo: CETESB, 1993. 86 p.

GLÓRIA, N. A. O uso agrícola de resíduos da indústria sucroalcooleira. In: CONGRESSO LATINO AMERICANO DE CIÊNCIA DO SOLO, 13., 1996, Águas de Lindóia. Anais... Águas de Lindóia: SBCS, 1996. CD-ROM.

KÖPPEN, W. M. Climatologia: con un studio de los climas de la terra. México: Fondo de Cultura Económica, $1948.478 \mathrm{p}$.
MARSCHNER, H. Mineral nutrition of higher plants. 2. ed. London: Academic Press, 1995. 889 p.

MIYAZAWA, M.; CHIERICE, G. O.; PAVAN, M. A. Amenização da toxicidade de alumínio às raízes do trigo pela complexação com ácidos orgânicos. Revista Brasileira de Ciência do Solo, Viçosa, MG, v. 16, n. 2, p. 209-215, 1992.

MONTEIRO, F. A.; RAMOS, A. K. B.; CARVALHO, D. D.; ABREU, J. B. R.; DAIUB, J. A. S.; SILVA, J. E. P.; NATALE, W. Cultivo de Brachiaria brizantha cv. Marandu em solução nutritiva com omissões de macronutrientes. Scientia Agrícola, Piracicaba, v. 52, n. 1, p. 35-141, 1995.

MORETI, D.; ALVES, M. C.; VALÉRIO FILHO, W. V.; CARVALHO, M. P. Atributos químicos de um Latossolo Vermelho sob diferentes sistemas de preparo, adubações e plantas de cobertura. Revista Brasileira de Ciência do Solo, Viçosa, MG, v. 31, n. 1, p. 167-175, 2007.

OLIVEIRA, P. P. A.; TRIVALIN, P. C. O.; OLIVEIRA, W. S.; CORSI, M. Fertilização com nitrogênio e enxofre na recuperação de pastagem de Brachiaria brizantha cv. Marandu em Neossolo quartzarênico. Revista Brasileira de Zootecnia, Viçosa, MG, v. 34, n. 4, p. 1121-1129, 2005.

PAVAN, M. A. Alumínio em alguns solos ácidos do Paraná. Relação entre o alumínio não trocável e solúvel com o pH, CTC, percentagem de saturação de $\mathrm{Al}$ e matéria orgânica. Revista Brasileira de Ciência do Solo, Viçosa, MG, v. 7, n. 1, p. 39-46, 1983.

PIRES, A. A.; MONNERAT, P. H.; MARCIANO, C. R.; PINHO, L. G. R.; ZAMPIROLLI, P. D.; ROSA, R. C. C.; MUNIZ, R. A. Efeito da adubação alternativa do maracujazeiro-amarelo nas características químicas e físicas do solo. Revista Brasileira de Ciência do Solo, Viçosa, MG, v. 32, n. 5, p. 7-16, 2008.

PLAZA, C.; SENESI, N.; POLO, A.; BRUNETTI, G.; GARCIA-GIL, J. C.; D'ORAZIO, V. Soil fulvic acid properties as a mean to assess the use of pig slurry amendment. Soil Tillage Research, Amsterdam, v. 74, n. 2, p. 79-190, 2003.

PRIMAVESI, A. C.; PRIMAVESI, O.; CORRÊA, L. A.; SILVA, A. G.; CANTARELLA, H. Lixiviação de nitrato em pastagens de coastcross adubada com nitrogênio. Revista Brasileira de Zootecnia, Viçosa, MG, v. 35, n. 3 , p. 683-690, 2006.

ROCHA, G. N.; GONÇALVES, J. L. M.; MOURA, I. M. Mudanças da fertilidade do solo e crescimento de um povoamento de Eucalyptus grandis fertilizado com biossólido. Revista Brasileira de Ciência do Solo, Viçosa, MG, v. 28, n. 4, p. 623-639, 2004. 
ROCHETTE, P.; ANGERS, D. A.; CÔTÉ, D. Soil carbon and nitrogen dynamics following application of pig slurry for the $19^{\text {th }}$ consecutive year: I. carbon dioxide fluxes and microbial biomass carbon. Soil Science Society of American Journal, Madison, v. 64, n. 4, p. 1389-1395, 2000.

ROSCOE, R.; NUNES, W. A. G. A; SAGRILO, E.; ORSUBA, A. A. Aproveitamento agrícola de resíduos de frigorífico como fertilizante orgânico sólido. Dourados: EMBRAPA Agropecuária Oeste, 2006. 30 p. (Boletim de Pesquisa e Desenvolvimento, 35).

SANTOS JUNIOR, J. D. G. Dinâmica de crescimento e nutrição do capim-marandu submetido a doses de nitrogênio. 2001. Dissertação (Mestrado em Agronomia) - Escola Superior de Agricultura Luiz de Queiroz. Universidade de São Paulo, Piracicaba.

SANTOS, A. B.; RABELO, R. R. Informações técnicas para a cultura do arroz irrigado no Estado do Tocantins. Santo Antônio de Goiás: Embrapa Arroz e Feijão, 2008. $136 \mathrm{p}$.

SANTOS, H. G. dos; JACOMINE, P. K. T.; ANJOS, L. H. C. dos; OLIVEIRA, V. A. de; OLIVEIRA, J. B. de; COELHO, M. R.; LUMBRERAS, J. F.; CUNHA, T. J. F. (Ed.). Sistema brasileiro de classificação de solos. $2^{\text {nd }} \mathrm{ed}$. Rio de Janeiro: Embrapa Solos, 2006. 306 p.

SCHERER, E. E.; BALDISSER, I. T.; NESI, C. N. Propriedades químicas de um latossolo vermelho sob plantio direto e adubação com esterco de suínos. Revista Brasileira de Ciência do Solo, Viçosa, MG, v. 31, n. 1, p. 123-131, 2007.

SILVA, F. C.; BOARETTO, A. E.; BERTON, R. S.; ZOTELLI, H. B.; PEXE, C. A.; BERNARDES, E. M. Efeito de lodo de esgoto na fertilidade de uma Argissolo Vermelho-Amarelo cultivado com cana-de-açucar. Pesquisa Agropecuária Brasileira, Brasília, v. 36, n. 5, p. 831-840, 2001.
SIMONETE, M. A.; KIEHL, J. C.; ANDRADE, C. A.; TEIXEIRA, C. F. A. Efeito do lodo de esgoto em um Argissolo e no crescimento e nutrição de milho. Pesquisa Agropecuária Brasileira, Brasília, v. 38, n. 10, p. 11871195, 2003.

SIDIRAS, N.; PAVAN, M. A. Influência do sistema de manejo do solo no seu nível de fertilidade. Revista Brasileira de Ciência do Solo, Viçosa, MG, v. 9, n. 3, p. 249-254, 1985.

SOUSA, D. M. G.; MIRANDA, L. N.; OLIVEIRA, S. A. Acidez do solo e sua correção. In: NOVAIS, R. F.; ALVAREZ, V. H. V.; BARROS, N. F.; FONTES, R. L. F.; CANTARUTTI, R. B.; NEVES, J. C. L. (Ed.). Fertilidade do solo. Viçosa, SBCS, 2007. p. 206-232.

TEDESCO, M. J.; SELBACH, I. C.; GIANELLO, C.; CAMARGO, F. A. O. Resíduos orgânicos e os impactos no ambiente. In: SANTOS, G. A.; SILVA, L. S.; CANELlAS, L. P.; CAMARGO, F. A. O. (Ed.). Fundamentos da matéria orgânica do solo: ecossistemas tropicais e subtropicais. Porto Alegre: Metrópole, 2008. p. 113-125.

THEODORO, V. C. A.; ALVARENGA, M. I. N.; GUIMARÃES, R. J.; SOUZA, C. A. S. Alterações químicas em solo submetido a diferentes formas de manejo do cafeeiro. Revista Brasileira de Ciência do Solo, Viçosa, MG, v. 27, n. 6, p. 1039-1047, 2003.

VIEIRA, R. F.; TANAKA, R. T.; TSAI, S. M.; PÉREZ, D. V.; SILVA, C. M. M. S. Disponibilidade de nutrientes no solo, qualidade de grãos e produtividade da soja em solo adubado com lodo de esgoto. Pesquisa Agropecuária Brasileira, Brasília, v. 40, n. 9, p. 919-926, 2005.

WERNER, J. C.; PAULINO, V. T.; CANTARELlA, H. Forrageiras. In: RAIJ, B. van; CANTARELLA, H.; QUAGGIO, J. A.; FURLANI, A. M. C. (Ed.). Recomendações de adubação e calagem para o Estado de São Paulo. Campinas: Instituto Agronômico, 1996. p. 263-273. (Boletim técnico, 100). 